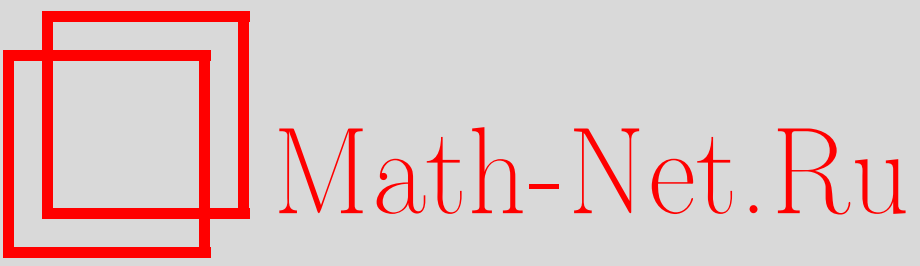

Б. М. Гуревич, А. А. Темпельман, О множествах временнь́х и пространственных средних для непрерывных функций на пространстве конфигураций, УМH, 2003, том 58, выпуск 2, 161-162

DOI: https://doi.org/10.4213/rm616

Использование Общероссийского математического портала Math-Net.Ru подразумевает, что вы прочитали и согласны с пользовательским соглашением

http: //www . mathnet.ru/rus/agreement

Параметры загрузки:

IP : 18.234 .197 .8

26 апреля 2023 г., 12:36:00 


\title{
О МНОЖКЕСТВАХ ВРЕМЕННЫ́ХХ И ПРОСТРАНСТВЕННЫХ СРЕДНИХ ДЛЯ НЕПРЕРЫВНЫХ ФУНКЦИЙ НА ПРОСТРАНСТВЕ КОНФИГУРАЦИЙ
}

\author{
Б. М. ГУРЕВИЧ, А. А. ТЕМПЕЛЬМАН
}

1. Рассмотрим пространство конфигураций $X=V^{\mathbb{Z}^{d}}$, где $|V|:=\operatorname{card} V<\infty, d \geqslant 1$, наделенное топологией прямого произведения, и группу $\tau$, состоящую из сдвигов $\tau_{t}, t \in \mathbb{Z}^{d}$, где $\left(\tau_{t} x\right)(s)=x(s+t), s \in \mathbb{Z}^{d}, x \in X, x: \mathbb{Z}^{d} \rightarrow V$. Зафиксировав последовательность конечных множеств $T_{n} \subset \mathbb{Z}^{d}$, положим для всякой функции $\varphi \in C\left(X, \mathbb{R}^{m}\right)$

$$
\left(A_{n} \varphi\right)(x)=\frac{1}{\left|T_{n}\right|} \sum_{t \in T_{n}} \varphi\left(\tau_{t} x\right), \quad x \in X, \quad n=1,2, \ldots
$$

Предел $\lim _{n \rightarrow \infty}\left(A_{n} \varphi\right)(x)=:(A \varphi)(x)$, если он существует, называется временны́ средним функции $\varphi$ в точке $x$ (роль “времени” здесь играет группа $\mathbb{Z}^{d}$ ). В далшнейшем мы всегда будем предполагать, что $T_{n}=\mathbb{Z}^{d} \cap \prod_{i=1}^{d}[0, n-1]$, хотя можно было бы рассматривать и более общие последовательности параллелепипедов (ср. с [1]). Для всякого $\alpha \in \mathbb{R}^{m}$ положим

$$
X_{\varphi, \alpha}=\{x \in X:(A \varphi)(x)=\alpha\} .
$$

Введем множество временни́х средних $A_{\varphi}=\left\{\alpha \in \mathbb{R}^{m}: X_{\varphi, \alpha} \neq \varnothing\right\}$ и множество пространственных средних $I_{\varphi}=\{\mu(\varphi), \mu \in \mathscr{I}\}$, где $\mu(\varphi):=\int \varphi d \mu$ и $\mathscr{I}$ - множество $\tau$-инвариантных вероятностных борелевских мер на $X$. Точка $x \in X$ назьвается типичной точкой меры $\mu$, если $(A \psi)(x)=\mu(\psi)$ для всех $\psi \in C\left(X, \mathbb{R}^{1}\right)$. Пусть $G_{\mu}$ - множество всех типичных точек меры $\mu$.

Введем на $X$ метрику $\rho=\rho_{\theta}$, положив $\rho_{\theta}(x, y)=\theta^{n(x, y)}, x \neq y$, где $\theta \in(0,1)$ и $n(x, y)$ - минимальное из тех $n$, для которых $x(t) \neq y(t)$ хотя бы при одном $t \in T_{n}$, и обозначим через $D_{\rho}(B)$ хаусдорфову размерность множества $B \subset X$, отвечающую метрике $\rho$ (все результаты этой статьи справедливы для гораздо более широкого класса метрик $\rho$, введенного в [2], см. также [3] и [4], в частности, для метрик Биллингсли, отвечающих гиббсовским мерам).

Теорема 1. Для всякой меры $\mu \in \mathscr{I}$ множество $G_{\mu}$ непусто и $D_{\rho_{\theta}}\left(G_{\mu}\right)=-h(\mu) / \ln \theta$, где $h(\mu)$ - әнтропия меры $\mu$.

Из первого утверждения теоремы получаем

СледствиЕ 1. Для всякой функции $\varphi \in C\left(X, \mathbb{R}^{m}\right)$ множества $A_{\varphi}$ и $I_{\varphi}$ совпадают, а потому $A_{\varphi}$ - выпуклое замкнутое множество.

Для случая $d=1$ непустота множества $G_{\mu}$ - это теорема Какутани (см. [5]), а его хаусдорфова размерность вычислена в [6] и [7]. Обобщение первого утверждения на случай произвольного $d \geqslant 1$ содержится в доказательстве леммы 3.1 из [3], там же доказано второе утверждение, но только для гиббсовских мер $\mu$.

2. Пусть $g \in C\left(X, \mathbb{R}^{m}\right)$. Мера $\mu \in \mathscr{I}$ называется $g$-равновесной (см. [1], [8]), если на ней достигается максимум функции $\nu \mapsto h(\nu)+\nu(g), \nu \in \mathscr{I}$. Совокупность $g$-равновесных мер обозначим через $\mathscr{E}(g)$.

Для всякого множества $B \subset \mathbb{R}^{m}$ будем обозначать через $\mathrm{cl} B$, int $B$ и ri $B$ соответственно его замькание, внутренность и относительную внутренность (см., например, [9]). Каждой функции $\varphi=\left(\varphi_{1}, \ldots, \varphi_{m}\right) \in C\left(X, \mathbb{R}^{m}\right)$ поставим в соответствие линейное подпространство $C_{\varphi} \subset C\left(X, \mathbb{R}^{1}\right)$, натянутое на $\varphi_{1}, \ldots, \varphi_{m}$. Пусть

$$
\mathscr{E}_{\varphi}=\bigcup_{g \in C_{\varphi}} \mathscr{E}(g), \quad E_{\varphi}=\left\{\nu(\varphi): \nu \in \mathscr{E}_{\varphi}\right\}
$$

Работа первого автора выполнена при частичной поддержке Российского фонда фундаментальных исследований (грант № 02-01-00158). 
ТеОрема 2. Для всякой функции $\varphi \in C\left(X, \mathbb{R}^{m}\right)$ выполняется включение $\operatorname{ri} I_{\varphi} \subseteq E_{\varphi}$.

ЗАмечАниЕ 1 . Разумеется, из теоремы 2 следует, что int $I_{\varphi} \subseteq E_{\varphi}$. Однако в некоторых ситуациях int $I_{\varphi}=\varnothing$, в то время как ri $I_{\varphi} \neq \varnothing$; это имеет место, например, в случае, когда функции $\varphi_{1}, \ldots, \varphi_{m}$ линейно зависимы.

СлЕДСТВИЕ 2. $\operatorname{cl}\left(\operatorname{ri} E_{\varphi}\right)=\operatorname{cl} E_{\varphi}=I_{\varphi}$.

3. По определению для всякого $\alpha \in E_{\varphi}$ найдется такая функция $g_{\alpha} \in C_{\varphi}$, что $\alpha=\mu_{\varphi, \alpha}(\varphi)$ для некоторой меры $\mu_{\varphi, \alpha} \in \mathscr{E}\left(g_{\alpha}\right)$.

TeOPema 3. Ecлu $\varphi \in C\left(X, \mathbb{R}^{m}\right) u \alpha \in E_{\varphi}, m_{\rho} D_{\rho}\left(X_{\varphi, \alpha}\right)=-h\left(\mu_{\varphi, \alpha}\right) / \ln \theta$.

В литературе имеется довольно много резултатов, родственных теореме 3 (см., например, [4], [10]-[15]). Однако, насколько мы можем судить, во всей своей общности она не вытекает из известных фактов даже для случая $d=1$.

При доказательстве теоремы 3 (и второго утверждения теоремы 1) используются результаты работы [3] и следующее утверждение.

Теорема 4. Для всякой меры $\mu \in \mathscr{I}$ существует такая последовательность әргодических гиббсовских мер $\mu_{n} \in \mathscr{I}$ с финитным потенциалом (т.е. марковских мер "растущего порядка"), что $\mu_{n}$ слабо сходится $\kappa \mu$ u $h\left(\mu_{n}\right)$ сходится $\kappa h(\mu)$.

Для $d=1$ это утверждение элементарно (см., например, [6]), для произвольного $d \geqslant 1$, но при дополнительном условии, что $\mu$ - гиббсовская мера, оно вытекает из [16] (см. также $[1 ; \S 16]$ ).

ЗАмеЧАнИЕ 2. Вероятно, большинство сформулированных выше результатов можно обобщить на динамические системы, удовлетворяющие условиям работ [10], [14], [15] (точнее, их обобщениям на случай $d>1$ ).

\section{СПИСОК ЛИТЕРАТУРЫ}

[1] Х.-О. Георги. Гиббсовские меры и фазовые переходы. М.: Мир, 1992. [2] А. А. Темпельман // Теория вероятн. и ее примен. 1999. Т. 44. № 3. С. 589-616. [3] В. M. Gurevich, A. A. Tempelman // J. Statist. Phys. 2002. V. 108. №5/6. P. 181-201. [4] A. A. Tempelman // J. Dynam. Control Systems. 2001. V. 7. № 4. P. 535-551. [5] J. C. Oxtoby // Ergodic Theory. Proc. Internat. Sympos., Tulane Univ., 1961. New York: Academic Press, 1963. P. 203-215. [6] H. Cajar // Lecture Notes in Math. 1981. V. 892. [7] E. Olivier // C. R. Acad. Sci. Paris Sér. I Math. 1999. V. 328. № 1. P. 13-16. [8] Д. Рюэль. Термодинамический формализм. Москва-Ижевск: РХД, 2002. [9] Р. Рокаффеллар. Выпуклый анализ. М.: Мир, 1973. [10] L. Barreira, B. Saussol, J. Schmeling // J. Math. Pures Appl. 2002. V. 81. P. 67-91. [11] A.-H. Fan, D.-J. Feng // J. Statist. Phys. 2000. V. 99. № 3/4. P. 813-856. [12] A.-H. Fan, D.- J. Feng, J. Wu // J. London Math. Soc. (2). 2001. V. 64. № 1. P. 229-244. [13] E. Olivier // Nonlinearity. 1999. V. 12. №6. P. 1571-1585. [14] F. Takens, E. Verbitski // Comm. Math. Phys. 1999. V. 203. № 3. P. 593-612. [15] F. Takens, E. Verbitski // Preprint, 2000. [16] A. Sokal // Comm. Math. Phys. 1982. V. 86. № 3. P. 327-336.

Московский государственньй университет им. М. В. Ломоносова; Университет штата Пенсильвания, США

E-mail: gurevich@mech.math.msu.su, arkady@stat.psu.edu
Принято редколлегией 03.02 .2003 